\title{
Research Paper \\ Comparing the Knee Joint Kinematic Parameters During Landing at Different Minutes of Soccer Game
}

\author{
*Abdolrasoul Daneshjoo ${ }^{1}$, Maryam Mohseni² (iD) \\ 1. Assistant Professor, Department of Biomechanics and Corrective exercise, East Tehran Branch, Islamic Azad University, Tehran, Iran. \\ 2. Master Degree in Sport Injuries and Corrective Exercise, East Tehran Branch, Islamic Azad University, Tehran, Iran.
}

\begin{tabular}{|l|l|}
$\begin{array}{c}\text { Use your device to scan } \\
\text { and read the article online }\end{array}$ \\
utes of Soccer Game (Persian)]. Journal of Sport Biomechanics. 2019; 5(1):2-13. https://doi.org/10.32598/biomechanics.5.1.1 \\
doi https://doi.org/10.32598/biomechanics.5.1.1
\end{tabular}

\section{(i) (8)}

Article Info:

Received: 10 Dec 2018

Accepted: 23 Apr 2019

Available Online: 01 Jun 2019

Key words:

Anterior cruciate ligament, Football, Landing, Dominant leg, Non dominant leg

\section{A B STRACT}

Objective Background The present research aimed to compare landing kinematics parameters at different minutes of a simulated soccer game.

Methods In total, 15 soccer players from the youth team of Sanay Mes of Kerman with records (2 years) in the Premier League of Iran voluntarily participated in this study. Their Mean \pm SD age and weight were 25.7 \pm 2.71 years and $77.30 \pm 7.78 \mathrm{~kg}$, respectively. The players followed the Bangsbo's fatigue protocol, i.e. a simulated soccer game. Then, every 15 minutes, the studied players performed two countermovement jumps, and we compared their landing kinematics parameters accordingly. Performances were recorded with 3 cameras, 120 HTZ (Sony A7S). Information extracted from Kinova and repeated-measures analysis of variance was used for data analysis.

Results The obtained results suggested significant differences between the investigated players' knee flexion at landing at minute 0 and minute 60 , and other durations $(P<0.05)$. Moreover, there were significant differences between dominant leg knee valgus at minutes 0,60 , and 105 , and other durations. No differences were detected between the non-dominant leg knee valgus at different minutes of the game.

Conclusion The obtained data revealed that minutes 0,60 , and 105 of a simulated soccer game are dangerous times for Anterior Cruciate Ligament ( $A C L$ ) injury in landing task. Moreover, the biomechanics of the dominant leg in the landing tasks demonstrate more changes than the non-dominant leg. Furthermore, ACL injury in the dominant leg is more frequent than the non-dominant one.

\section{Extended Abstract}

\section{Introduction}

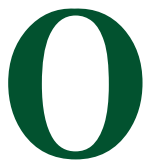

ne of the mechanisms of lower limb injury is landing. This movement can create a force as large as 2 to 12 times the bodyweight; the skeletal system must moderate such mechanical shock [1]. Repeated increasing impact forces during landing provide the basis for structural damage to the soft tissue around the joint [2].
Numerous factors influence the landing mechanics of football players. Some of the most essential causes of serious knee injuries are gender, age, and fitness level. Besides, some physiological factors, such as fatigue, increased knee valgus angle, and decreased knee flexion during landing, might affect them. The superior leg is stronger than the non-superior one; however, the Anterior Cruciate Ligament (ACL) injury is greater in this leg, compared to the nonsuperior leg [2]. Researchers reported that landing with decreased lower limb and knee flexion angles causes more significant anterior tibial shear force; thus, they suggested

\section{* Corresponding Author:}

Abdolrasoul Daneshjoo, PhD.

Address: Department of Biomechanics and Corrective exercise, East Tehran Branch, Islamic Azad University, Tehran, Iran.

Tel: +98 (912) 2061034

E-mail: phdanesh@yahoo.com 
that athletes flex their trunk and knee joints more frequently during landing [8].

No study was found to examine the kinematic parameters of the lower limb during landing at different minutes of a football play. Therefore, this study aimed to compare the kinematic parameters of lower extremities during landing at different minutes of a soccer game.

\section{Participants and Methods}

In total, 15 youth players of FC Mes of Kerman Industry with the Mean \pm SD age of $17.55 \pm 1.12$ years, the weight of $62.21 \pm 7.22 \mathrm{~kg}$, and height of $175.43 \pm 7.88 \mathrm{~cm}$ volunteered to participate in the current study. The players performed the Benzo Buddies Fatigue Protocol, i.e. similar to soccer. Then, they made two reverse jumps every 15 minutes. After recording the video with three cameras (Sony A7S), we extracted the landing data through the Kinovea software. Using SPSS, we compared the kinematic performance of the study subjects in different minutes of a football game. We also used the repeated measures Analysis of Variance (ANOVA) to compare time intervals (Table 1).

\section{Results}

Paired comparisons of $0,15,30$, and 75 minutes with other measurements were statistically significant $(\mathrm{P}<0.05)$; however, other comparisons were not significant $(\mathrm{P}>0.05)$. Table 2 compares the mean scores of repeated measures ANOVA of the maximum valgus-varus knee angle during landing between the superior legs.

\section{Discussion}

This study investigated the risk factors of ACL injuries during landing at different minutes of a soccer game. The obtained results suggested that knee flexion before initiating the game was significantly less than that of the other time intervals. After this time, knee flexion has been relatively high during landing. After a half-time break, the knee flexion decreased again in the $60^{\text {th }}$ minute (after the second half started). There was less flexion in the knee joint be-

Table 1. Comparing the repeate d measures ANOVA values of the knee's deepest joint flexion during landing

\begin{tabular}{|c|c|c|c|c|c|c|}
\hline Source of Changes & Statistics & Sum of Squares & df & Mean of Squares & $\mathbf{F}$ & $\mathbf{P}$ \\
\hline & Greenhouse-gieser & 17087.017 & 3.734 & 4575.737 & 562.19 & 0.001 \\
\hline \multirow[t]{3}{*}{ Score } & Heine-felt & 17087.017 & 5.262 & 3247.506 & 562.19 & 0.001 \\
\hline & Lower bound & 17087.017 & 1.000 & 17087.017 & 562.19 & 0.001 \\
\hline & Greenhouse-gieser & 12229.025 & 52.280 & 233.915 & - & - \\
\hline \multirow[t]{2}{*}{ Error } & Heine-felt & 12229.025 & 73.662 & 166.015 & - & - \\
\hline & Lower bound & 12229.025 & 14.000 & 873.502 & - & - \\
\hline
\end{tabular}

Table 2. Comparing the repeated measures ANOVA values of the maximum valgus-varus knee angle during landing between the superior legs

\begin{tabular}{ccccccc}
\hline Source of Changes & Statistics & Sum of Squares & df & Mean of Squares & F & P \\
\hline \multirow{2}{*}{ Score } & Greenhouse-gieser & 1573.965 & 3.244 & 485.143 & 0.898 & 0.456 \\
& Heine-felt & 1573.965 & 4.339 & 362.777 & 0.898 & 0.477 \\
& Lower bound & 1573.965 & 1.000 & 1573.965 & 0.898 & 0.359 \\
& Greenhouse-gieser & 371.15302 & 41.160 & 371.774 & - & - \\
Error & Heine-felt & 371.15302 & 292.53 & 287.143 & - & - \\
& Lower bound & 371.15302 & 14.000 & 026.1093 & - & - \\
\hline
\end{tabular}


fore starting the first and second halves, compared to other times. Therefore, this was due to lower body temperature and muscle minutes before the start of each half of the game. This result is in line with Mohr's research [3].

\section{Conclusion}

The achieved results revealed that the knee flexion process during the soccer game had an upward course. In other words, the first few minutes of each half had the lowest knee flexion rate during landing; accordingly, the odds of ACL injury incidence was higher during these intervals.

According to the previous hypothesis, the highest valgus angle of the superior leg occurred at 0 and 60 minutes of a football play. This finding indicates a relationship between lower knee flexion and increased valgus angle. Furthermore, the early minutes of each half-time are potentially dangerous for ACL injury. Moreover, fatigue affected the kinematics of the superior leg during landing; i.e. in the 105th minute of play, the superior leg knee has increased knee valgus and enhanced the risk of ACL injury. The nonsuperior leg of the players appeared to be less likely to have an ACL injury. This might be because of the lack of knee valgus at any time interval and that a knee varus angle has always occurred. This might have resulted from non-superior leg neuromuscular adaptations.

\section{Ethical Considerations}

\section{Compliance with ethical guidelines}

All subjects voluntarily participated in the study and signed the written consent form. IR. IAUETB.96141 Article Code of Ethics

\section{Funding}

This article is taken from Ms. Maryam Mohseni's Master of MS. in Physical Education and Sport Sciences, Islamic Azad University, East Tehran Branch.

\section{Authors' contributions}

The whole process of editing the article was the responsibility of the first author, and Ms. Maryam Mohseni was responsible for conducting tests and preparing the raw data.

\section{Conflicts of interest}

The authors declared no conflict of interest.

\section{Acknowledgements}

The authors thank the officials of the Kerman Mess Sports Club and the players participating in the tests. 


\title{
مقايسه يارامترهاي كينماتيكى مفصل زانو حين فرود در دقايق مختلف بازى فوتبال
}

\author{
“(1) 'عبدالرسول دانشجو' (1)، مريم محسنى \\ ا. .استاديار كروه بيومكانيك وحركات اصلاحي، واحد تهران شرق، دانشكاه آزاداسلامى، تهران، ايران.

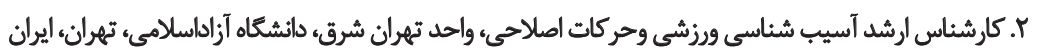

\begin{abstract}
حكيد
هدف هدف از اين تحقيق مقايسه يارامترهاى كينمائيكى اندام تحثاني بازيكنان فوتبال حين فرود در دقايق مختلف بازى فوتبال بود.

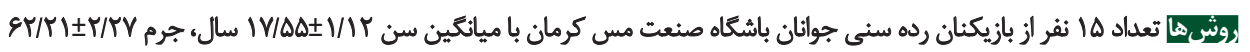

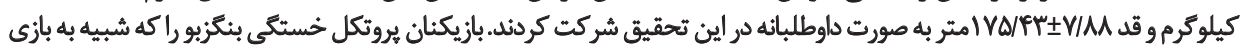

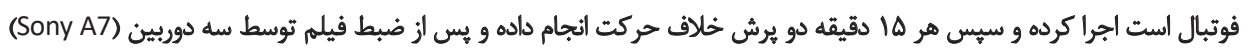

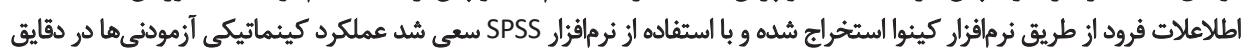

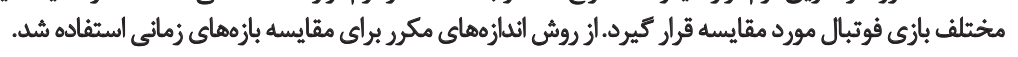

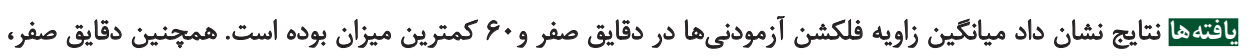

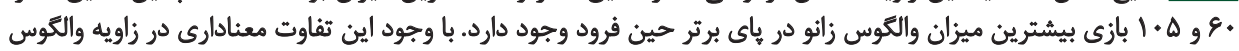

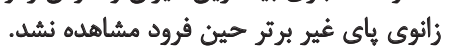

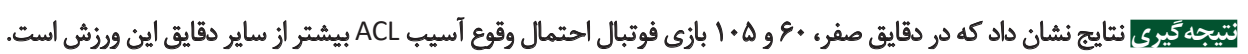

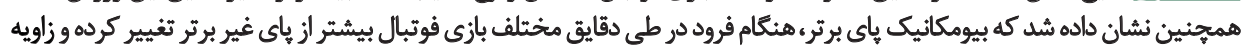

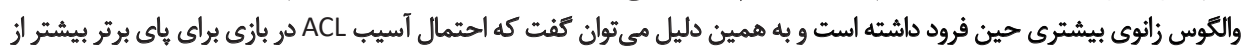

اطلاعات مقاله: تاريخ دريافت: 19

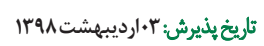

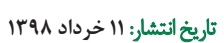

كليدواروهها:

رباط صليبى قدامى،

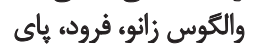
برثر، هاي غير برثر

بازمهاى زمانى فوتبال مي متواند عملكرد بازيكنان را در دري

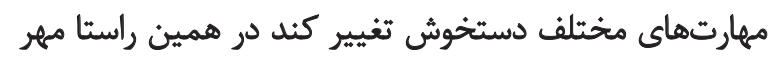

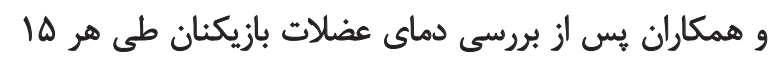

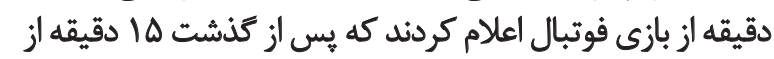

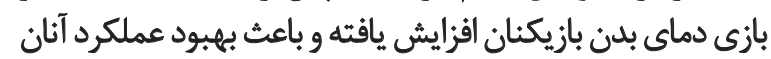

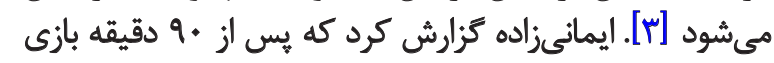

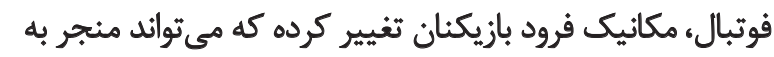

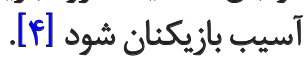

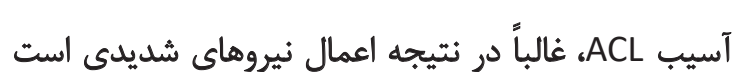

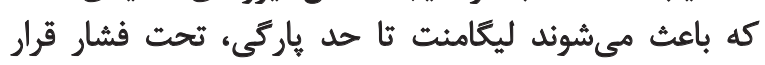

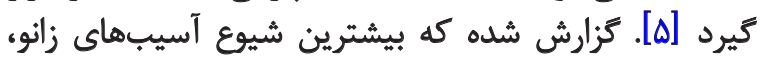

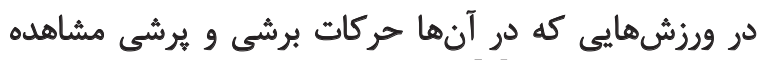

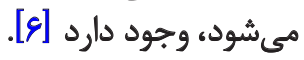
سربازى، كزارش كرد كه تفاوت معنادارى بين بارامترهاى

مقدمه

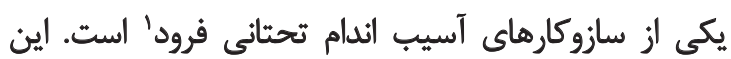

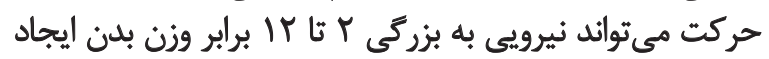

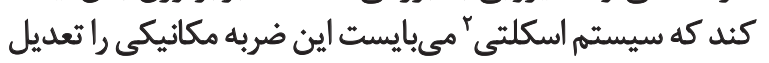

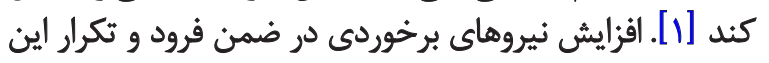

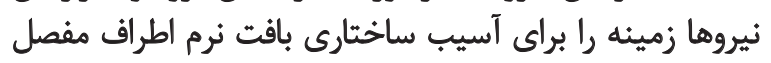

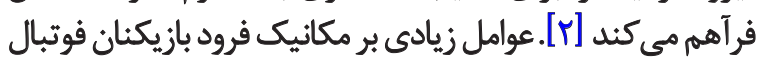

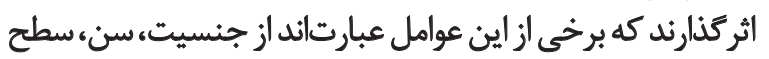

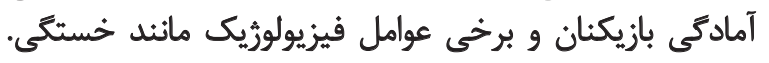

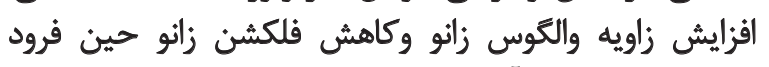

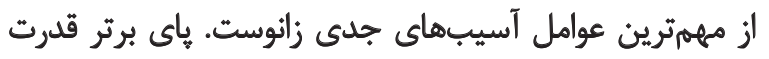

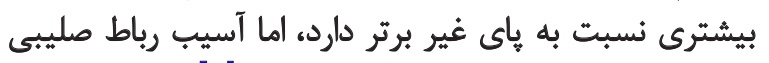

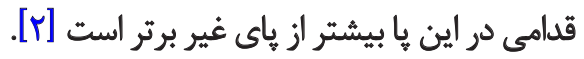

1. Landing

2. Skeletal system

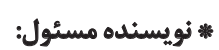

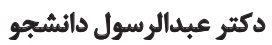

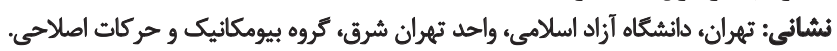

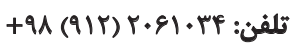
يست الكترونيكي: phdanesh@yahoo.com 


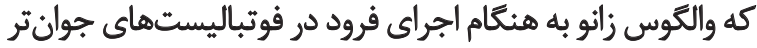

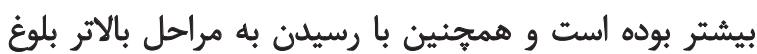

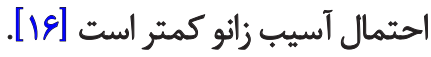

جنسيت و ياي برثر و غير برثر بر ظرفيت جذب شوك حين عين

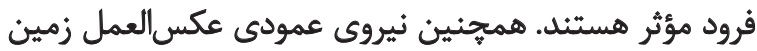

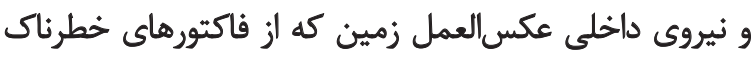

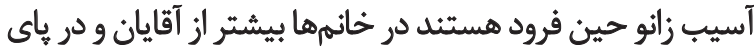

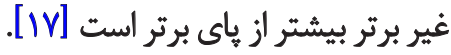
باوجوداين، تحقيقى يافت نشد كه يار امترهاي كينماتيكى اندام

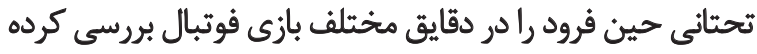

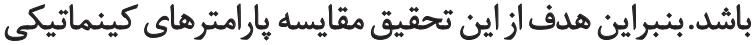

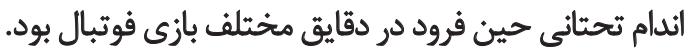

روشُشَّى

تحقيق حاضر به صورت بثروهشى ـ كاربردى بود. يارامترهاى

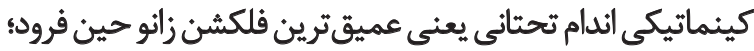

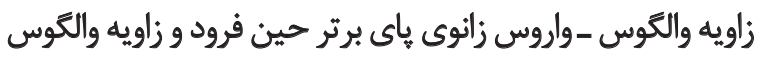

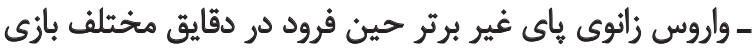

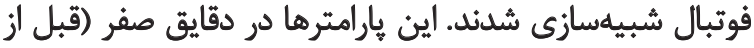

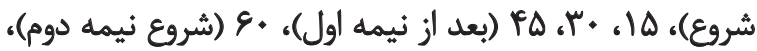

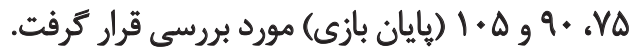
جامعه آمارى اين تحقيق شامل بازيكنان رده سنى جوانان باشكاه

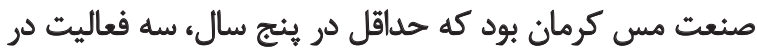

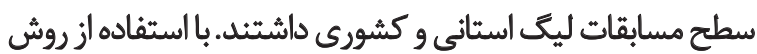

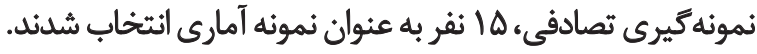

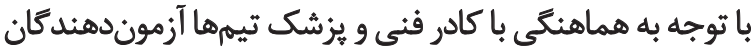

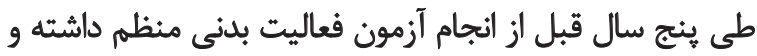

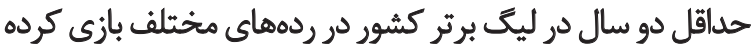

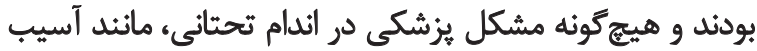
ACL

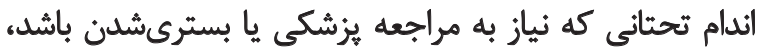

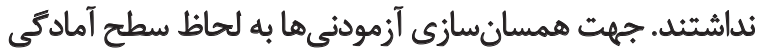

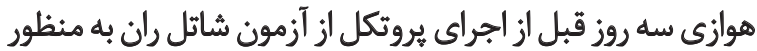
بررسى VO

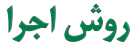

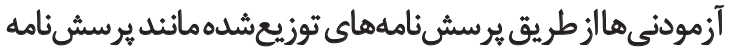

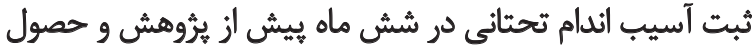

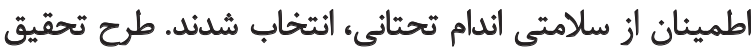

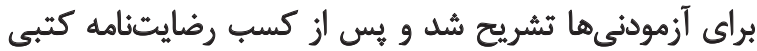
براى شركت در آزمون از آنها دعوت بـ به عمل آمد آدم.

با توجه به نياز حركت برش خلاف حركت، به هماهنكى

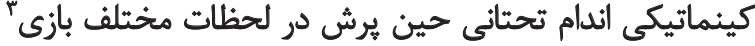

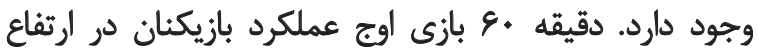

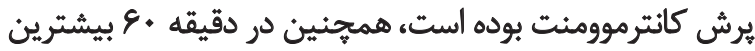
جابهجايى مفصل زائو، بيشترين سرعت اكسنتريك و و بيشترين

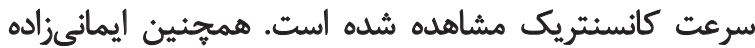

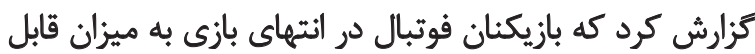

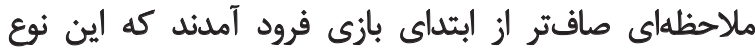

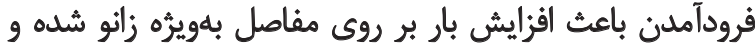

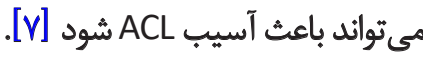
محققين كزارش كردند كه هنكام فرود با زاويه فلكشن كمتر

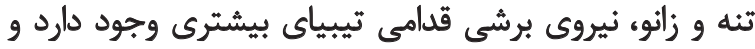

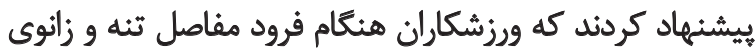

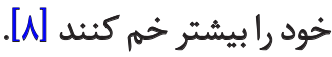
زنان بسكتباليست به هنكام فرود، والكوس زانوى بيشترى دارئد

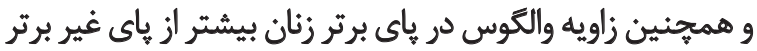

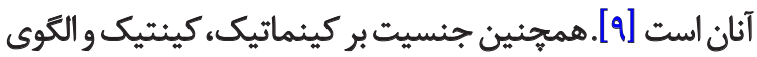

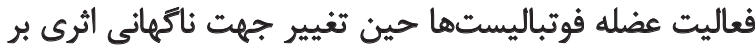
ايجاد تفاوت كينماتيكى ندارد [.1]

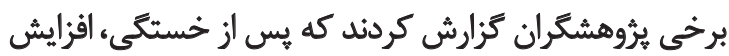

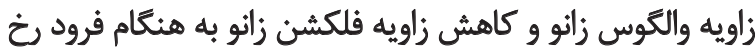

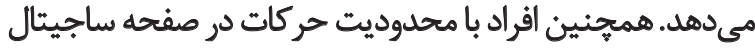

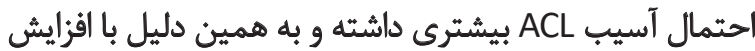

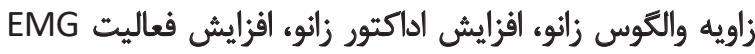

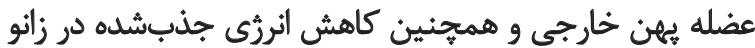

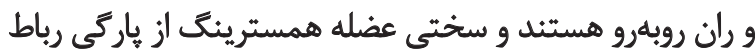
صليبى قدامي حين فرود بيشكيرى مي وكند [111] خستىى شكمى مىتواند احتمال آسيب ACL را به به وسيله

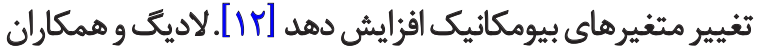

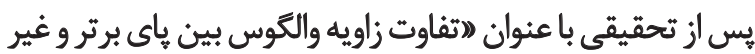

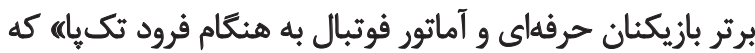

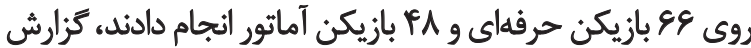

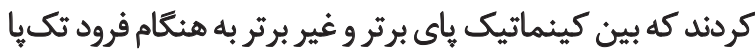

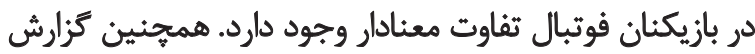

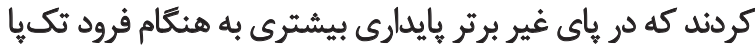

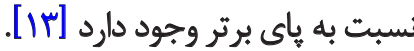

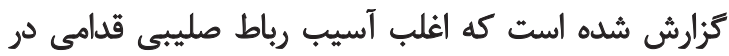

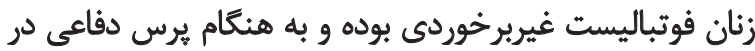

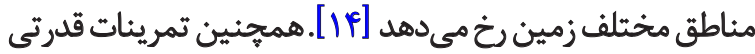

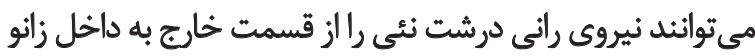

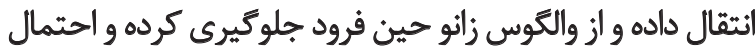

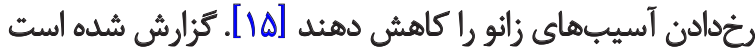

3. Different minutes 


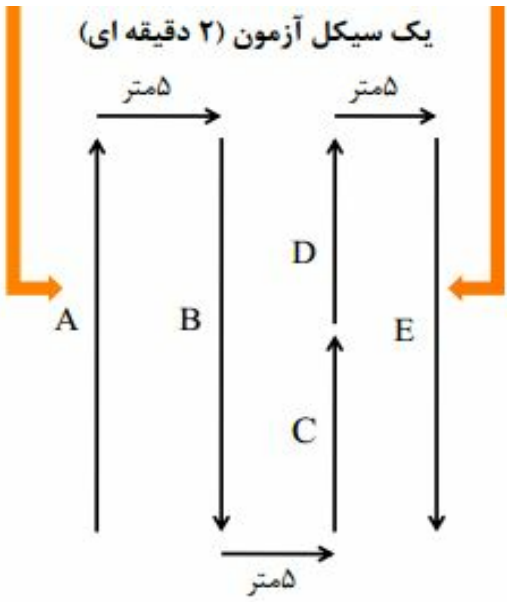

$$
\begin{aligned}
& \text { A } \\
& \text {. مت متر دريبل با توب از ميان مخروطها. } \\
& \text { •ه متر دويدن به سمت عقب } \\
& \text { TA متر دويدن زير بيشينه } \\
& \text { D } \\
& \text { TA متر دويدن با حداكثر سرعت } \\
& \text { E } \\
& \text { •ه متر قدم زدن }
\end{aligned}
$$

مجله بيومكانيك وزنث

دقيقه باقى مىماند به عنوان دوره استراحت محاسبه شد. ملاكى

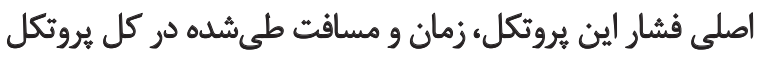

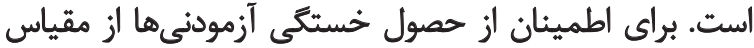

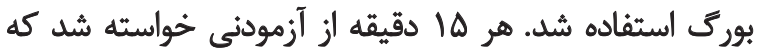

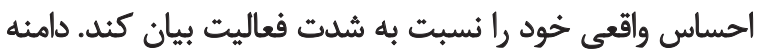

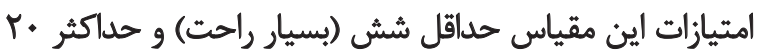

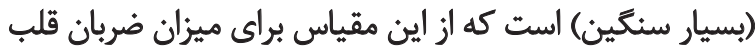

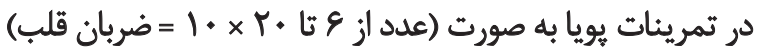

$$
\text { استفاده مىشود. }
$$

با توجه به هدف تحقيق، آزمودنىها ها الديقات دقيقه با استفاده

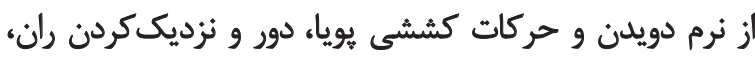

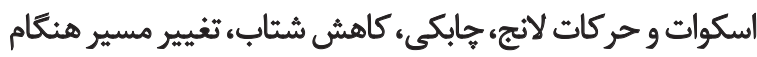

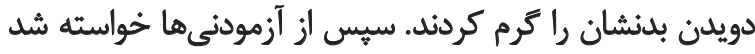

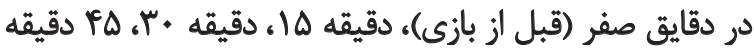

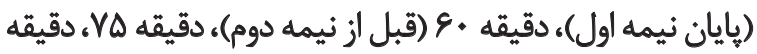

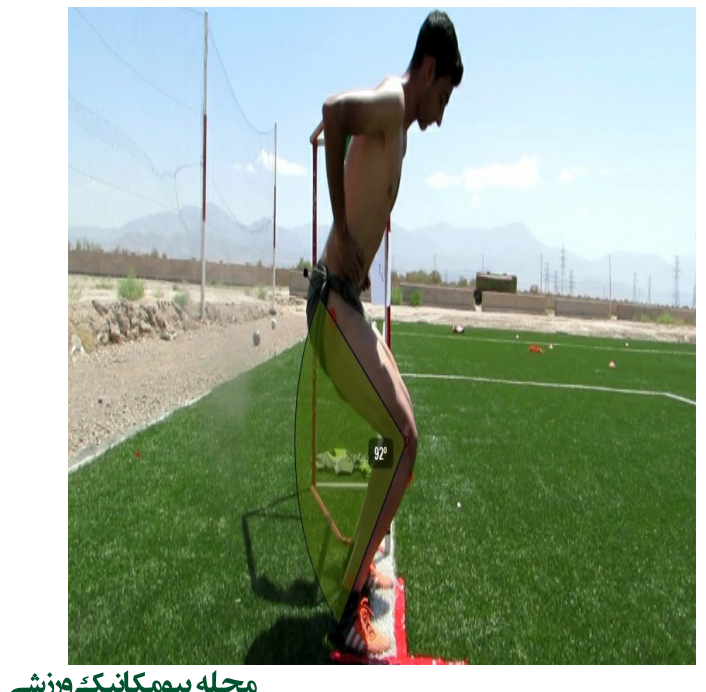

تصوير r. بيشترين زاويه فلكشن مفصل زانو حين فرود
تصوير ا.مراحل اجراى آزمون فوتبال

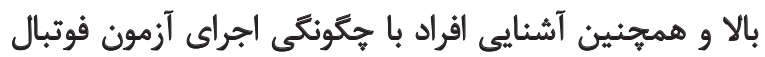

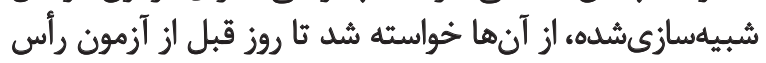

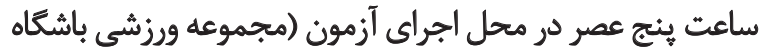

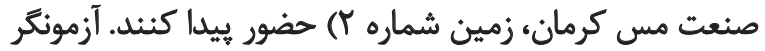

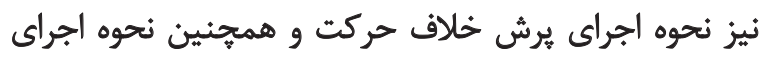

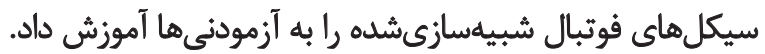

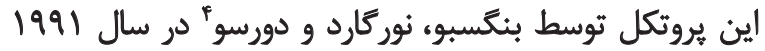

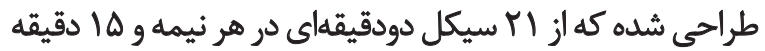

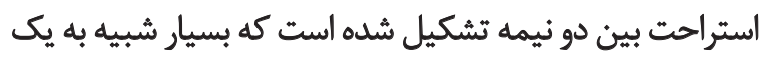
بازى فوتبال است (تصوير شماره ()).

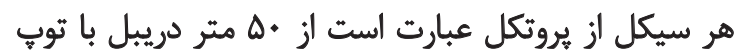

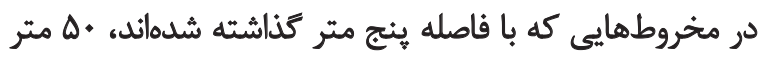

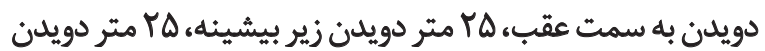

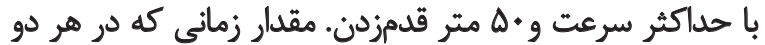

4. Bangsbo, Nogard, Dorso

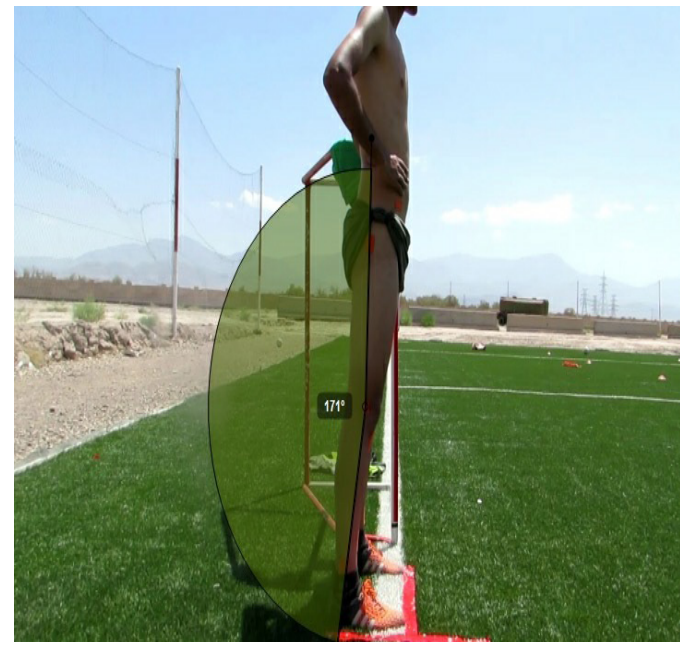

مجله بيومكانيك وزنش

تصوير T. شروع هيرش و بررسى ميزان زاويه مفصل زائو در صفحه ساجيتال 


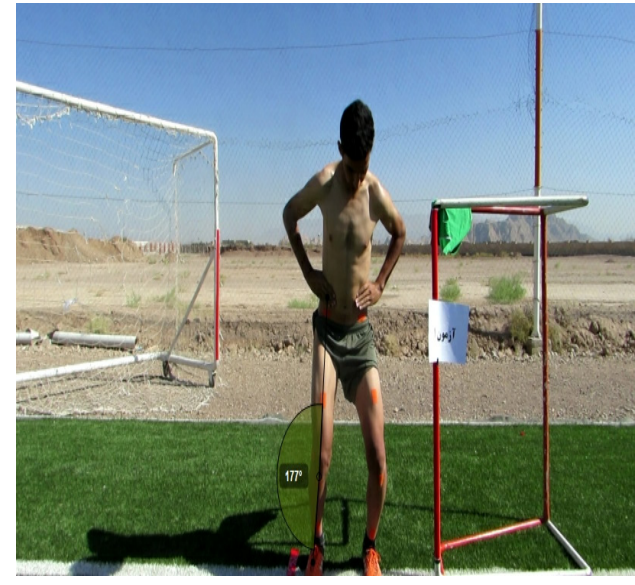

مجله بيومكانيك وزنث

تصوير ه. زاويه والثكوس - واروس مفصل زانو حين فرود بازه زمانى را مشخص كرد. به منظور بررسى عميقترين فلكشن فين

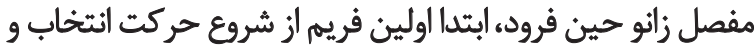

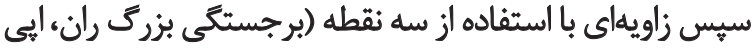

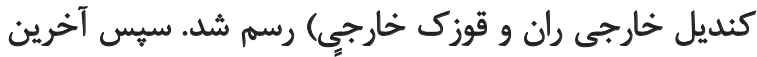

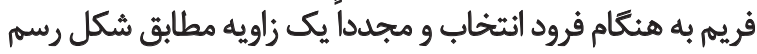

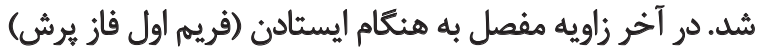

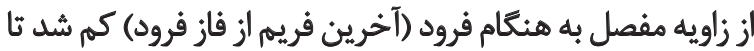
عميقترين زاويه مفصل زانو به دست آيد.

عميث ترين فلكشن = زاويه زائو (ايستادن) ـ بيشترين زاويه

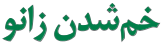

جهت بررسى زاويه والكوس -واروس ابتدا اولين فريم از حركت

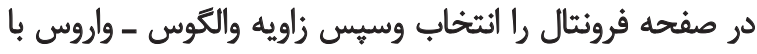

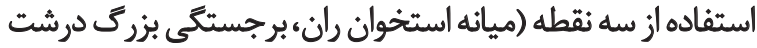

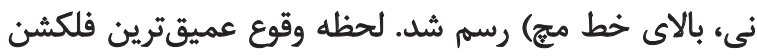

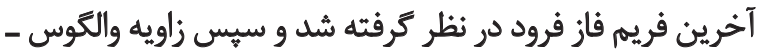

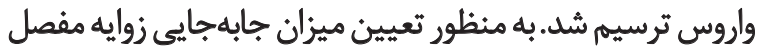

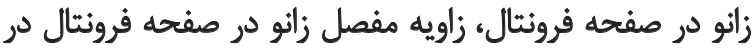

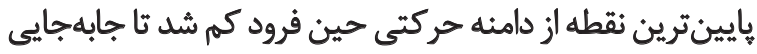

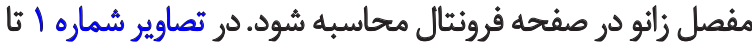
ه مراحل و شيوه اجراى بثروهش نشان داده شدهاند.

$$
\text { روش آمارى }
$$

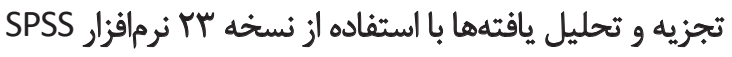

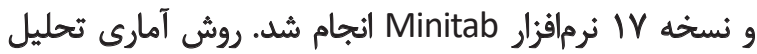

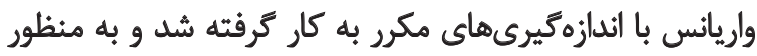

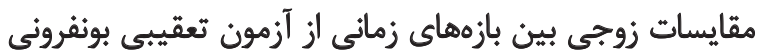

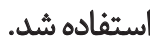

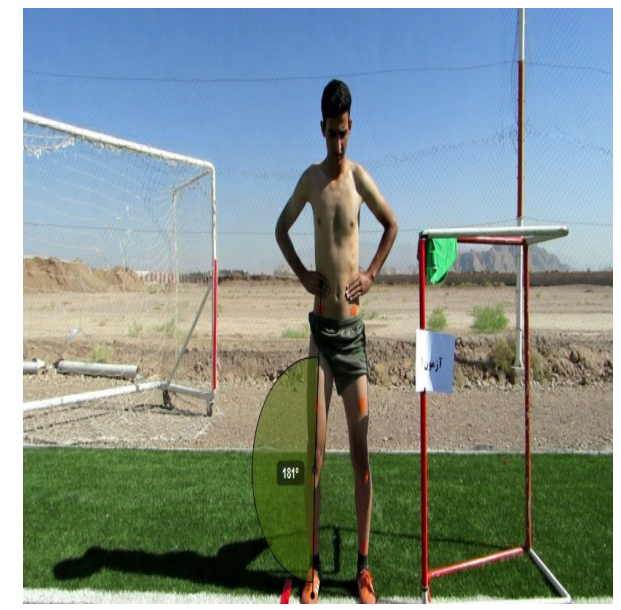

مجله بيومكانيك وزنش

$$
\text { تصوير F. زاويه والكوس - واروس مفصل زانو قبل از شروع يرش }
$$

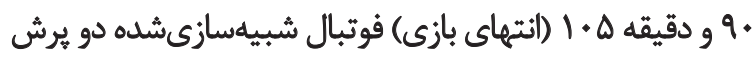

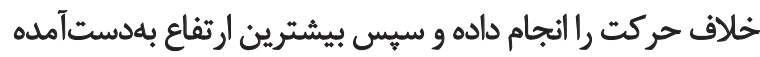

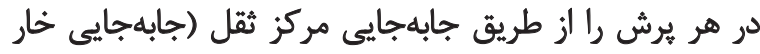

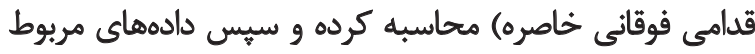

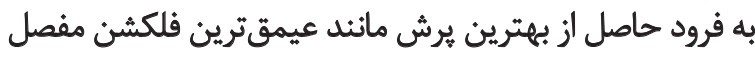

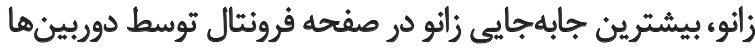

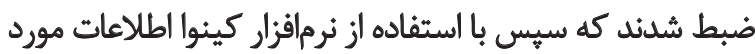
نياز آناليز واستخراج شد.

در مجموع شش نشائكر بر روى نقاط آناتوميكى هر فرد كذاشته

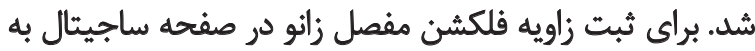

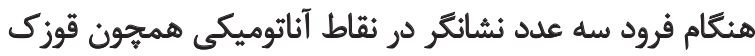

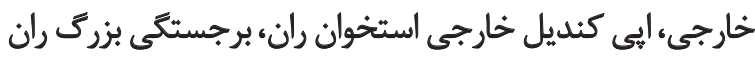

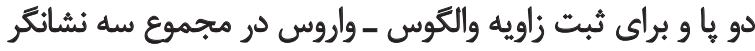

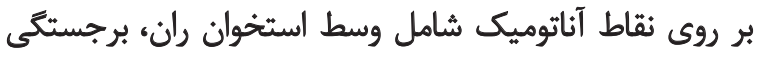

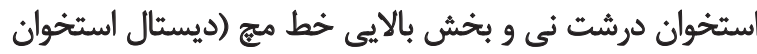

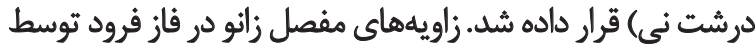

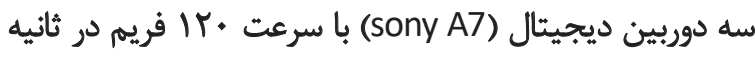

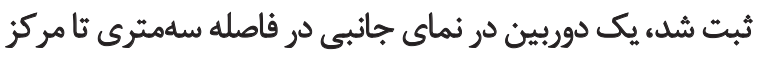

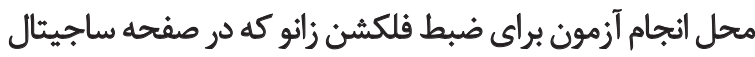
رخ مى دهد قرار داده شد و دو دوربين ديكر (sony A7) در نمائ

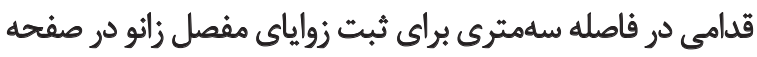

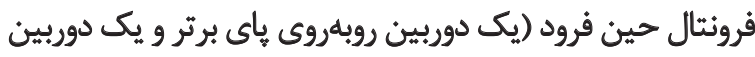
روباروى پاى غير برتر) قرار داده شد.

برى بالارفتن سرعت فيلمبردارى و ايجاد فريمهاى بيشتر از

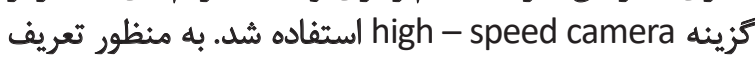

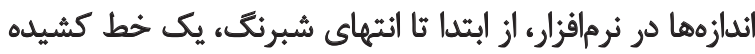

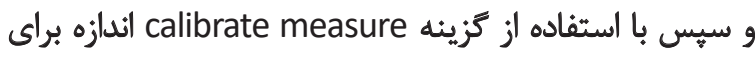

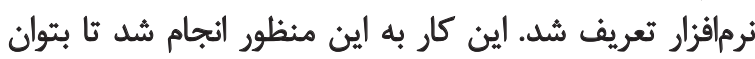

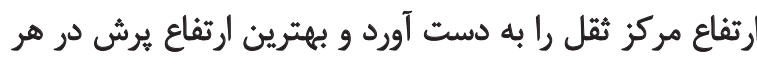




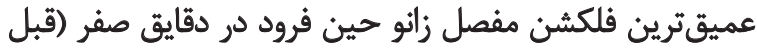

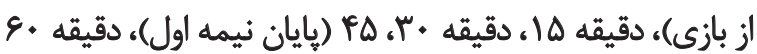

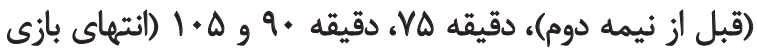

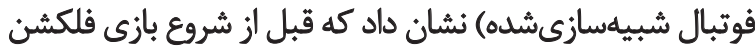

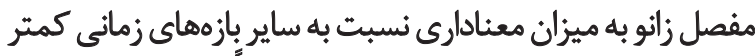

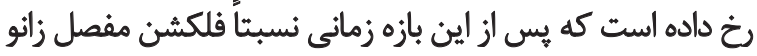

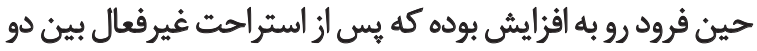

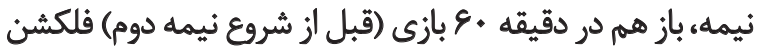

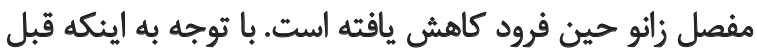

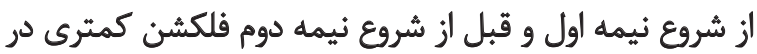

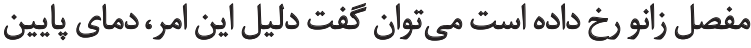

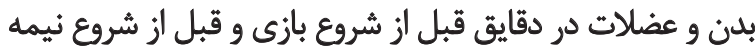

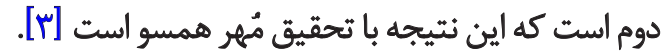
فلكشن مفصل زانو از مهمثرين مكانيسمهاي منجر به به آسيب

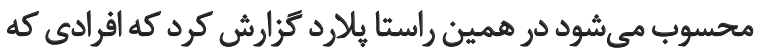

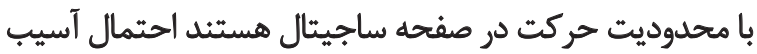

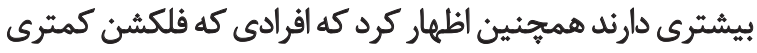

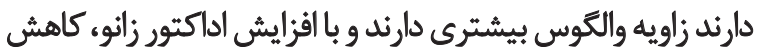

توصيف ويرٔكى هاى بالينى و جمعيتشناختى آزمودنى ها در

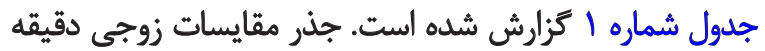

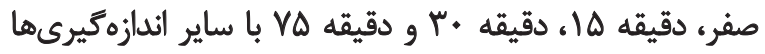

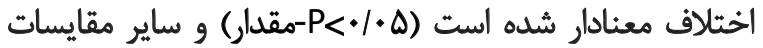

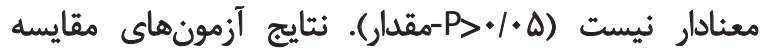

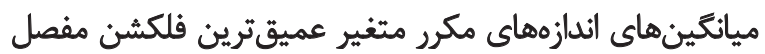

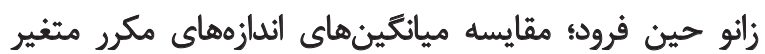

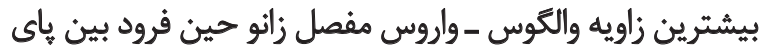

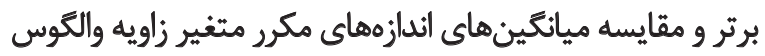

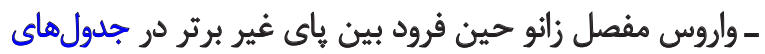

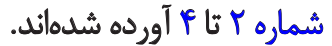

ب

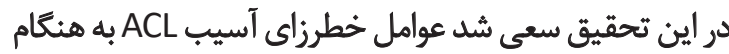

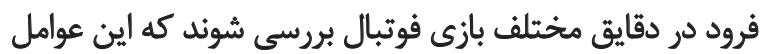

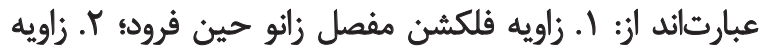

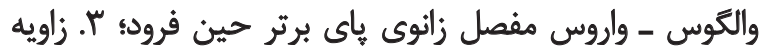

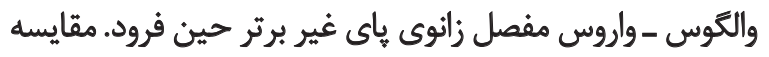
جدول ا. توصيف ويرُكى هاى جمعيتشناختى آزمودنى مها

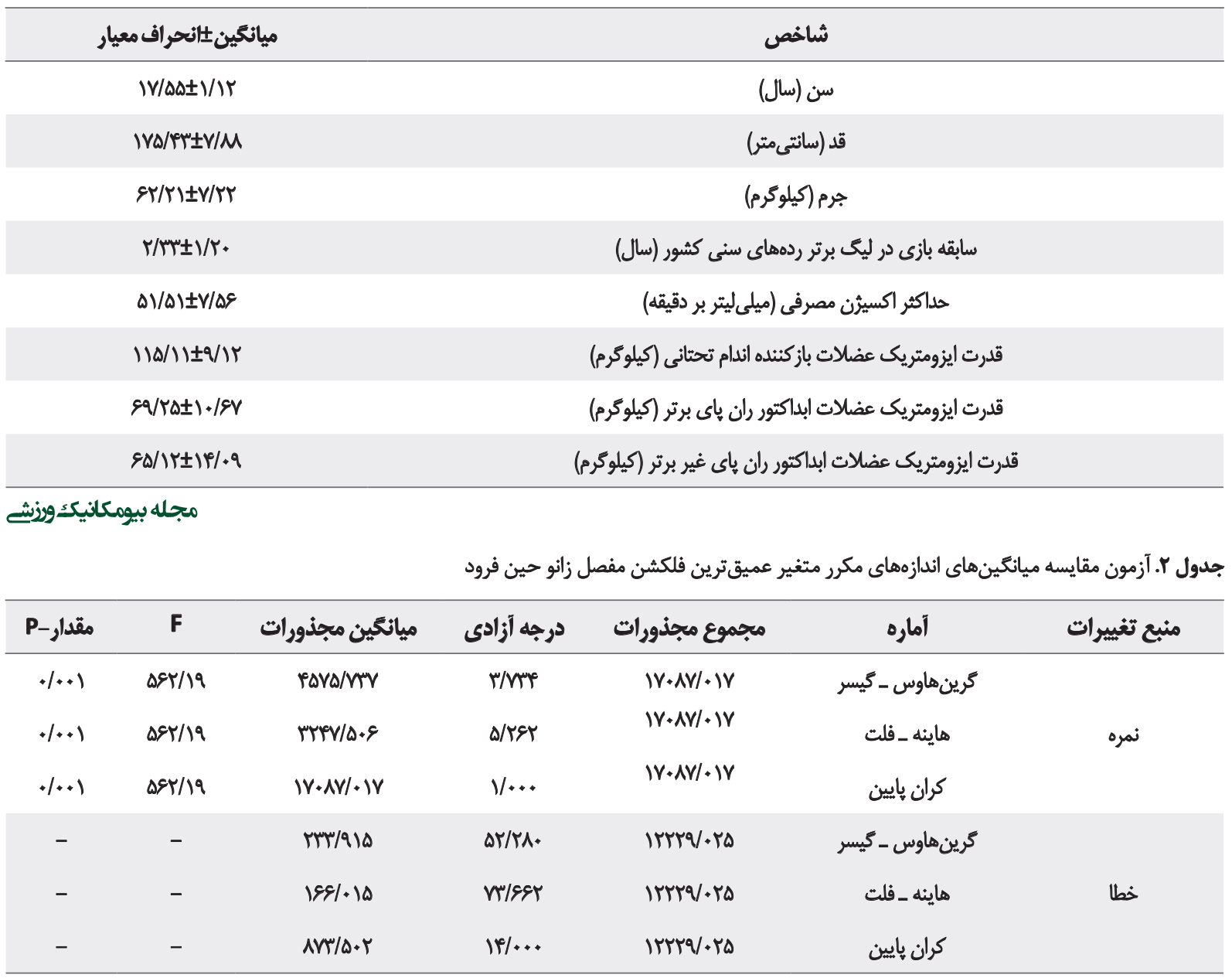

مجله بيومكانيك وزنثـ 
جدول ऍ. آزمون مثايسه ميانكينهاي اندازههاي مكرر متغير بيشترين زاويه والتكوس - واروس مفصل زانو حين فرود بين باي برتر

\begin{tabular}{|c|c|c|c|c|c|c|}
\hline Pa مقدار & $\mathbf{F}$ & ميانغين مجذورات & درجه آزادى & مجموع مجذورات & آماره & منبع تغييرات \\
\hline - MAOS &.$/ 124$ & PNA/IFT & r/MPT & $10 V T / a c \Delta$ & كرينهاوس - كيسر & \\
\hline.$/ F W$ & - /14 & rgT/WN & F/rTq & $\mid \Delta V_{T} / Q Q \Delta$ & هائه ـ فلت & نمره \\
\hline.$/ r \Delta q$ & - 114 & $10 V / / Q 8 \Delta$ & $18 \ldots$ & $10 V^{2} / 98 \Delta$ & كران هايين & \\
\hline - & - & 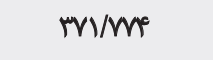 & Fi/le. & $|Q T \cdot r / m|$ & كرينهاوس ـ كيسر & \\
\hline - & - & rAV/IRT & QH/Tar & $|Q T \cdot T / M V|$ & هاينه _فلت & خطا \\
\hline - & - & $1.94 / .48$ & $|f| \ldots$ & $\left|\Delta T^{\prime}+r / T r\right|$ & كران بايين & \\
\hline
\end{tabular}

مجله بيومكانيك ورنش

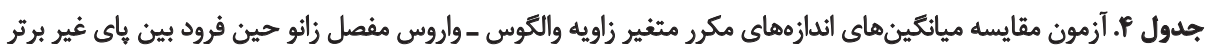

\begin{tabular}{|c|c|c|c|c|c|c|}
\hline Pa Palر & $\mathbf{F}$ & ميانكين مجذورات & دوجه آزادى & مجموع مجذورات & آماره & منبع تغييرات \\
\hline . /FAS &.$/ 144$ & FAD/IFH & $r / M \mu T^{2}$ & $\mid \Delta V^{L} / Q \Omega \Delta$ & كرينهاوس - كيسر & \\
\hline . /ew & - /AM & TETIM & P/MTq & $1 \Delta \gamma^{2} / \Omega \& \Delta$ & هاينه ـ قلت & نمره \\
\hline . /roq & .1144 & $\mid \Delta V T / Q P Q$ & $11 \ldots$ & IONT/QSA & كران بايين & \\
\hline - & - & $\Delta P+/ 1 \Delta C$ & PA/TTI & MPATEREA & كرينهاوس - كيسر & \\
\hline - & - & $P \cdot r / q 1 \Delta$ & $8 \cdot M P I$ & TPATT/rea & هاينه _ فلت & خطا \\
\hline- & - & IVAT/REY & $\mid f / \ldots$ & Trame/rea & كران هايين & \\
\hline
\end{tabular}

مجله بيومكانيك ونشث

اكرجه تحقيقات ديكرى هستند كه نشان مي إدهند بإيان

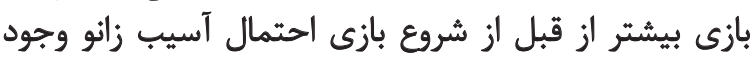

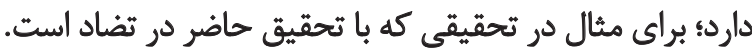

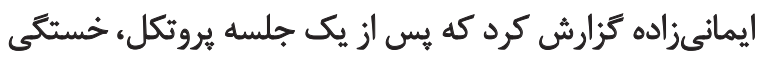

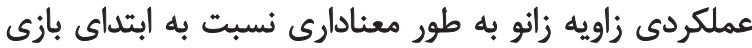

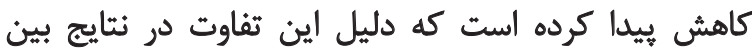

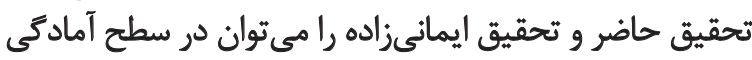

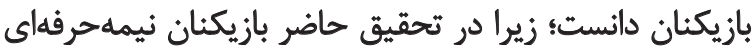

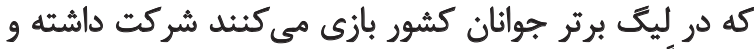

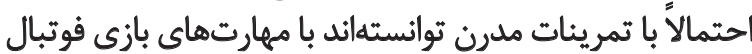

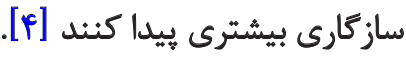

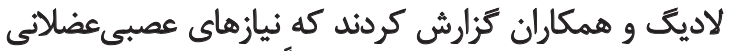

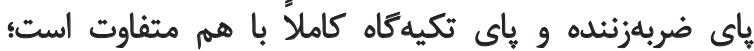

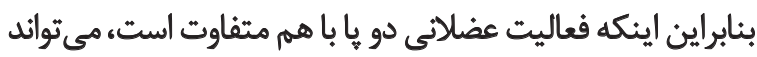

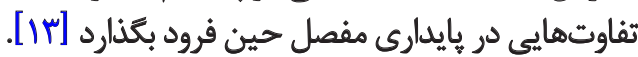

در دقايق ابتدايى نيمه اول و دوم عملكرد بازيكنان حين

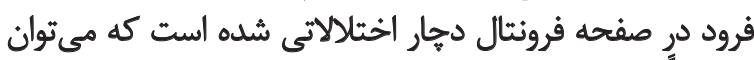

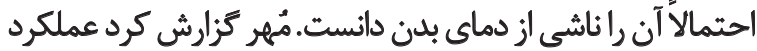

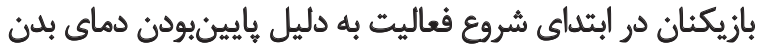

انرزى جذب شده در زانو و ران و افزايش الكترومايوكرافى عضله

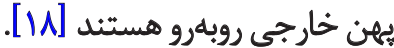

هتلر اعلام كرد كه بيشترين خطر آسيب ACL به هنكام

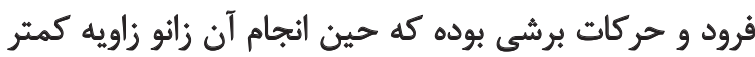

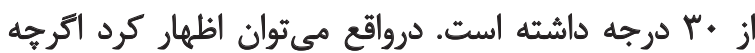

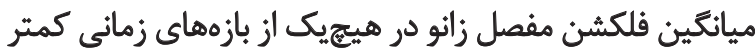

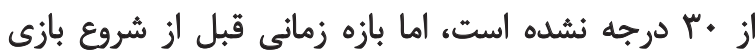

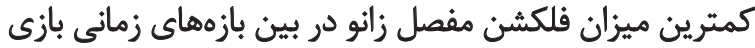

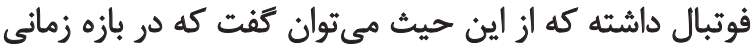

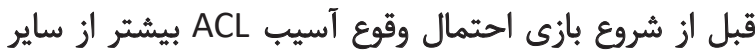

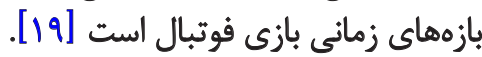

نتايج تحقيق حاضر با تحقيق مورفى و ديكين در تضاد

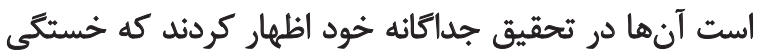

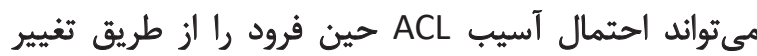

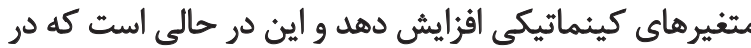

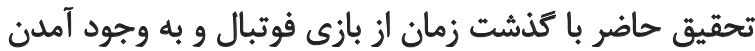

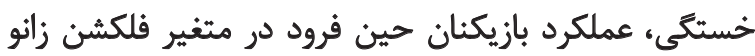

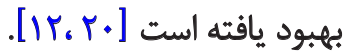




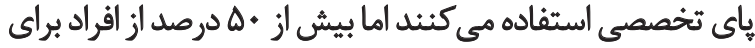

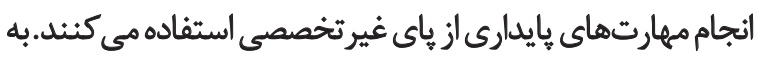

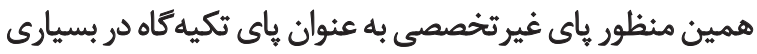

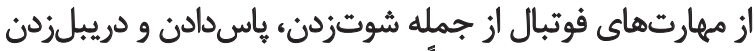

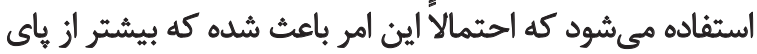

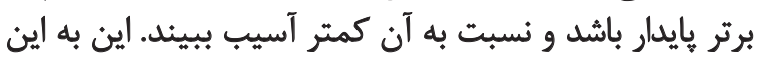

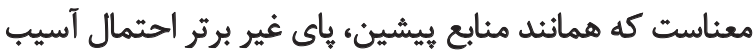

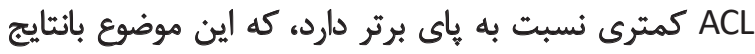

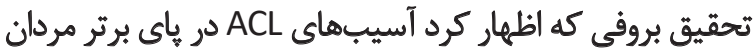

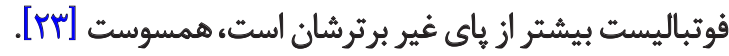

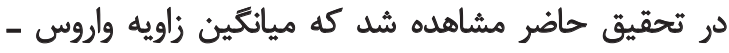

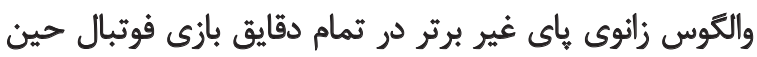

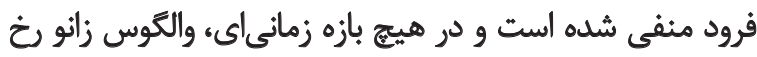

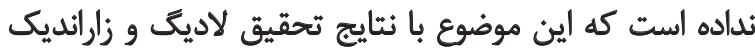

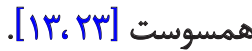

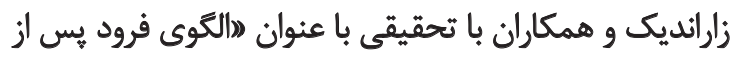

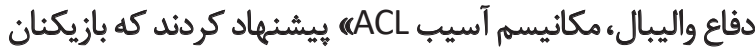

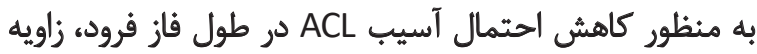

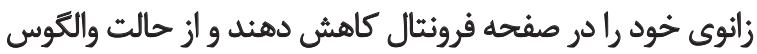

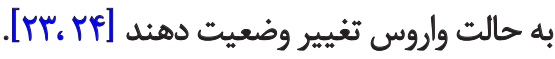

تمرينات متفاوتى مى توانند منجر به كاهش والكوس زانو حين

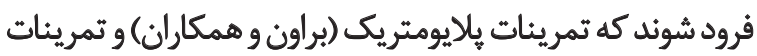

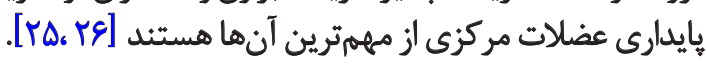

لاديگ وهمكاران بِ إز تحقيقى كه بر روى هو ع بازيكن

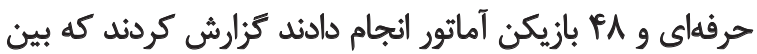

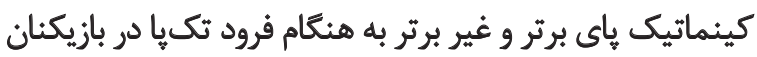

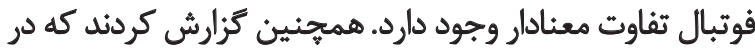

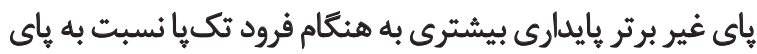

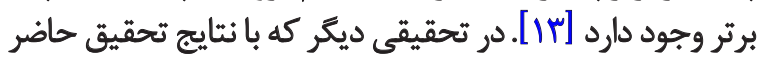

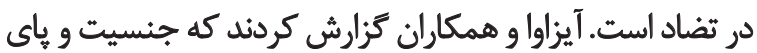

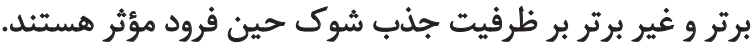

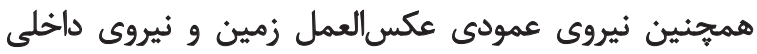

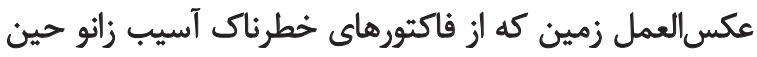

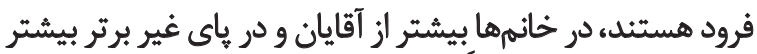

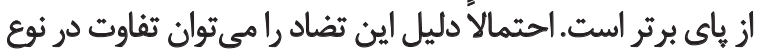

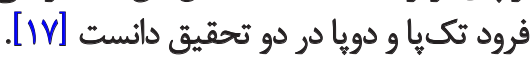

\section{نتيجه نيرى نهايى}

نتايج كلى بررسىها نشان داد كه روند فلكشن زانو در حين

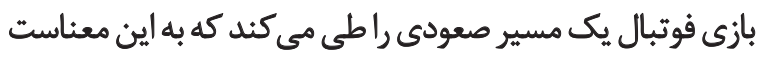

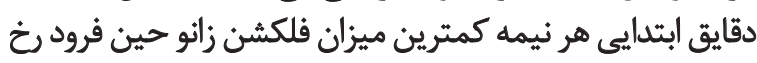

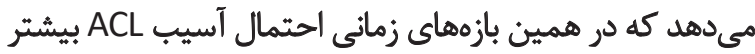

و عضلات كمتر از ساير زمانهاى بازي بوده است كه با كذشت

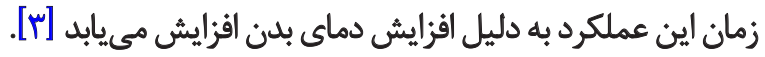

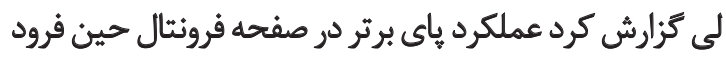

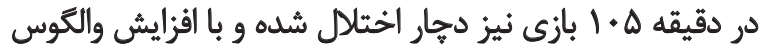

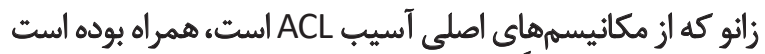

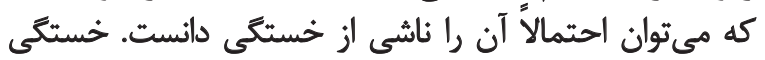

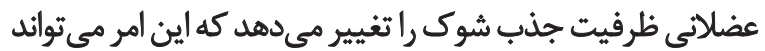

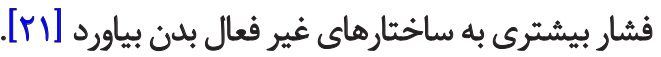
تحقيقاتى در زميئه خستكى و مكانيسم فرود انجام شده است

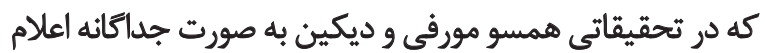

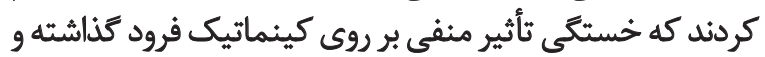

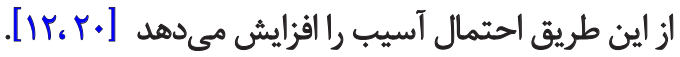

در تحقيقى ديكر با عنوان لاثأثير خستكى عملكردى ويثر ائه

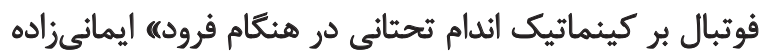

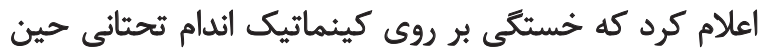

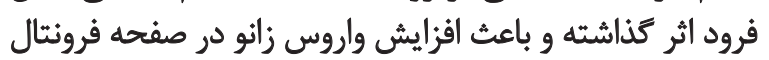

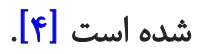

بروفى و همكاران با تحقيقى با عنوان "اخستكى ابداكتورهاى زئي

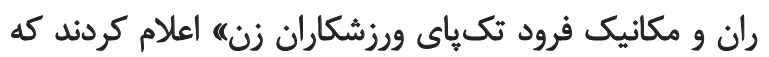

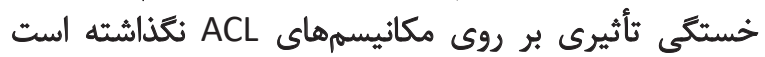
كه البته دليل آن را الحتمال وجئ رود ابداكشن زائو حين فرود نكانيت

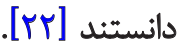

با توجه به فرضيات يُروهش حاضرمبنى بر وجود بيشترين زاويه

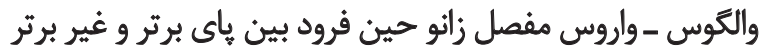

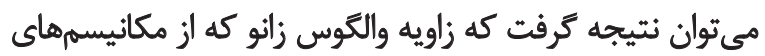

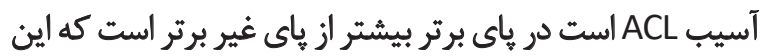

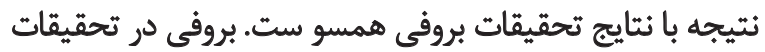

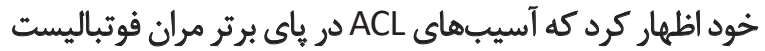

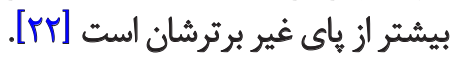
هماجنين در تحقيقى همسو با تحقيق حاضر لاديك و همكاران

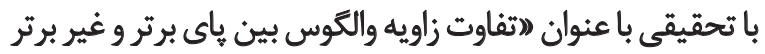

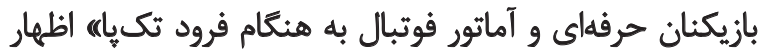

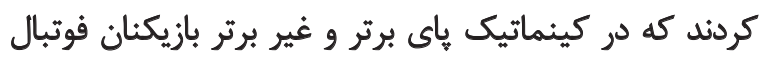
تفاوتهايى وجود دارد [1 [ir].

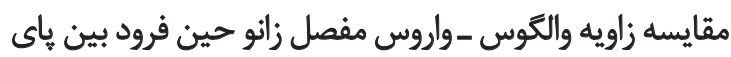

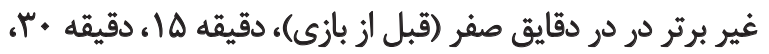

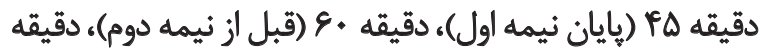

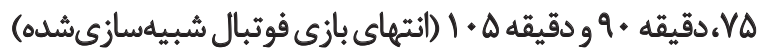

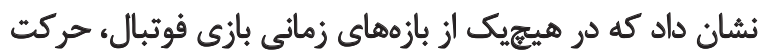

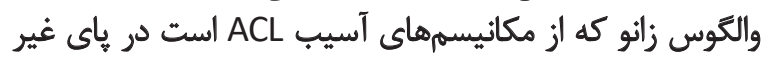

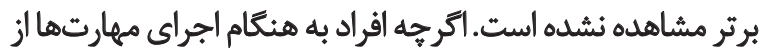


است. همائند فرضيه قبل بيشترين زاويه والكوس هائ برتر در

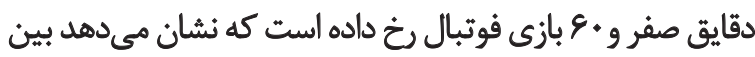

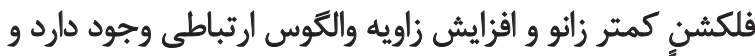

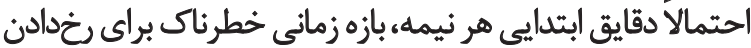

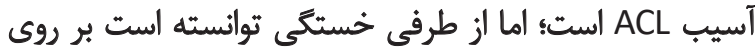

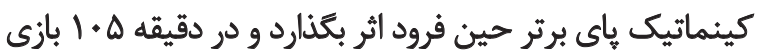

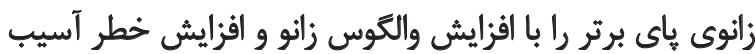

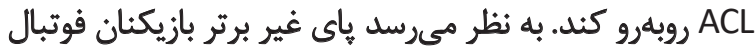

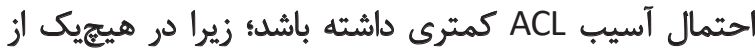

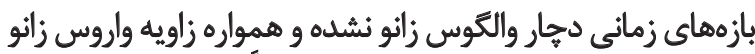

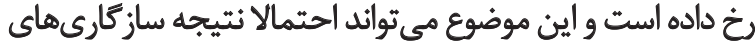
عصبى عضلاتى ياي غير برثر باشد.

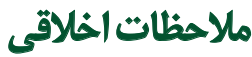

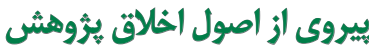

تمامى آزمودنىها داوطلبانه در اين يُروهش شركت كردند

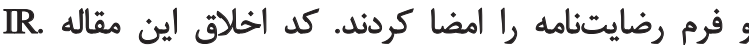

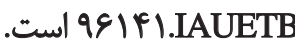

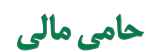

مقاله حاضر بركرفته از واياينامه كارشناسى ارشد خانم مريم

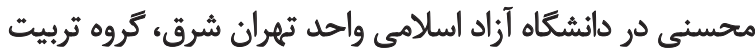
بدنى و علونم ورزشى است.

$$
\text { مشاركت نويسند مكان }
$$

تمامى مراحل تنظيم مقاله به عهده نويسنده اول بوده است و

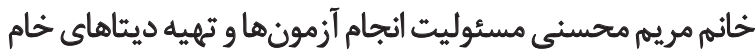

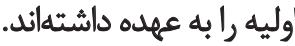

$$
\text { تعارض مثاقع }
$$

بنا به اظهار نويسندكان اين مقاله تعارض منافع ندارد.

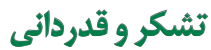

بدينوسيله نويسندكان از مسئولين باشكاه فرهنكيىورزشيى

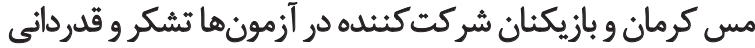

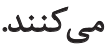




\section{References}

[1] McLean SG, Huang X, Su A, Van Den Bogert AJ. Sagittal plane biomechanics cannot injure the $\mathrm{ACL}$ during sidestep cutting. Clinical Biomechanics. 2004; 19(8):838-28. [DOI:10.1016/j.clinbiomech.2004.06.006] [PMID]

[2] Wu HW, Liang KH, Lin YH, Chen YH, Hsu HC. Biomechanics of ankle joint during landing in counter movement jump and straddle jump. Paper presented at: IEEE $35^{\text {th }}$ Annual Northeast Bioengineering Conference. 3-5 April 2009; Boston, MA, USA. [DOI:10.1109/NEBC.2009.4967733]

[3] Mohr M, Krustrup P, Bangsbo J. Fatigue in soccer: A brief review. Journal of Sports Sciences. 23(6):593-9. [DOI:10.1080/02640410400021286] [PMID]

[4] Imanizadeh S. [The effect of lasting on kinematics of lower extremities during landing (Persian)] [MSc. thesis]. Kerman: Shahid Bahonar University of Kerman; 2013. https://ganj-old.irandoc.ac.ir/articles/670184

[5] Chappell JD, Herman DC, Knight BS, Kirkendall DT, Garrett WE, Yu B. Effect of fatigue on knee kinetics and kinematics in stop-jump tasks. The American Journal of Sports Medicine. 2005; 33(7):1022-9. [DOI:10.1177/0363546504273047] [PMID]

[6] Girard O, Nybo L, Mohr M, Racinais S. Plantar flexor neuromuscular adjustments following match-play football in hot and cool conditions. Scandinavian Journal of Medicine \& Science in Sports. 2015; 25(Supp 1):154-63. [DOI:10.1111/sms.12371] [PMID]

[7] Sarbazi MA. [Comparison of the kinematic parameters of the lower extremity during the jump of the countermoniton at different moments of the simulated soccer game (Persian)] [MSc. thesis]. Tehran: East Tehran Branch, Islamic Azad University; 2017.

[8] Rajabi R, Mohammadpour Sh. [The relationship of kinematics of trunk and knee in sagittal plane with anterior tibia shear force during single leg landing (Persian)]. Scientific Journal of Kurdistan University of Medical Sciences. 2014; 19(2):47-56. [DOI:10.22102/19.2.47]

[9] Ford KR, Myer GD, Hewett TE. Valgus knee motion during landing in high school female and male basketball players. Medicine \& Science in Sports \& Exercise. 2003; 35(10):1745-50. [DOI:10.1249/01. MSS.0000089346.85744.D9] [PMID]

[10] Sigward SM, Powers CM. The influence of gender on knee kinematics, kinetics and muscle activation patterns during side-step cutting. Clinical Biomechanics. 2006; 21(1):41-8. [DOI:10.1016/j.clinbiomech.2005.08.001] [PMID]

[11] Rössler R, Junge A, Chomiak J, Dvorak J, Faude O. Soccer injuries in players aged 7 to 12 years: A descriptive epidemiological study over 2 seasons. The American Journal of Sports Medicine. 2016; 44(2):309-17. [DOI:10.1177/0363546515614816] [PMID]

[12] Murphy E. Abdominal fatigue and lower extremity kinematics during a drop landing in females. Bellingham, WA: Western Washington University; 2015. https://cedar.wwu.edu/cgi/viewcontent. cgi?article $=1404 \&$ context $=$ wwuet

[13] Ludwig O, Simon S, Piret J, Becker S, Marschall F. Differences in the dominant and non-dominant knee valgus angle in junior elite and amateur soccer players after unilateral landing. Sports. 2017; 5(1):pii:E14. [DOI:10.3390/sports5010014] [PMID] [PMCID]

[14] Kaneko S, Sasaki Sh, Hirose N, Nagano Y, Fukano M, Fukubayashi T. Mechanism of anterior cruciate ligament injury in female soccer players. Asian Journal of Sports Medicine. 2017; 8(1):e38205. [DOI:10.5812/ asjsm.38205]
[15] Czasche MB, Goodwin JE, Bull AM, Cleather DJ. Effects of an 8-week strength training intervention on tibiofemoral joint loading during landing: A cohort study. BMJ Open Sport \& Exercise Medicine. 2018, 4(1):e000273. [DOI:10.1136/bmjsem-2017-000273] [PMID] [PMCID]

[16] Read PJ, Oliver JL, De Ste Croix MBA, Myer GD, Lloyd RS. Landing kinematics in elite male youth soccer players of different chronologic ages and stages of maturation. Journal of Athletic Training. 2018; 53(4):372-8. [DOI:10.4085/1062-6050-493-16] [PMID] [PMCID]

[17] Aizawa J, Hirohata K, Ohji S, Ohmi T, Yagishita K. Limb-dominance and gender differences in the ground reaction force during single-leg lateral jump-landings. Journal of Physical Therapy Science. 2018; 30(3):387-92. [DOI:10.1589/jpts.30.387] [PMID] [PMCID]

[18] Pollard CD, Sigward SM, Powers CM. Limited hip and knee flexion during landing is associated with increased frontal plane knee motion and moments. Clinical Biomechanics. 2010; 25(2):142-6. [DOI:10.1016/j. clinbiomech.2009.10.005] [PMID] [PMCID]

[19] Hettler J, Myklebust G. Avoiding the failed ACL: How to prevent ACL tears before they occur. In: Marx R, editor. Revision ACL Reconstruction. New York, NY: Springer; 2014. [DOI:10.1007/978-1-4614-0766-9_2]

[20] Dickin DC, Johann E, Wang H, Popp JK. Combined effects of drop height and fatigue on landing mechanics in active females. Journal of Applied Biomechanics. 2015; 31(4):237-43. [DOI:10.1123/jab.2014 0190] [PMID]

[21] Frankel VH, Nordin M. Biomechanics of bone. In: Nordin M, Franke $\mathrm{VH}$, editors. Basic Biomechanics of the Musculoskeletal System. Philadelphia: Lippincott Williams \& Wilkins; 2002.

[22] Brophy R, Silvers HJ, Gonzales T, Mandelbaum BR. Gender influences: The role of leg dominance in $\mathrm{ACL}$ injury among soccer players. British Journal of Sports Medicine. 2010; 44(10):694-7. [DOI:10.1136/ bjsm.2008.051243] [PMID]

[23] Brown TN, Palmieri-Smith RM, McLean SG. Comparative adaptations of lower limb biomechanics during unilateral and bilateral landings after different neuromuscular-based $\mathrm{ACL}$ injury prevention protocols. The Journal of Strength \& Conditioning Research. 2014; 28(10):2859-71. [DOI:10.1519/JSC.0000000000000472] [PMID]

[24] Zahradnik D, Jandacka D, Farana R, Uchytil J, Hamill J. Landing patterns after block in volleyball: Aplication for ACL injury. Paper presented at: $33^{\text {rd }}$ International Conference on Biomechanics in Sports, June 29-July 3, 2015; Poitiers, France.

[25] Myer GD, Ford KR, McLean SG, Hewett TE. The effects of plyometric versus dynamic stabilization and balance training on lower extremity biomechanics. The American Journal of Sports Medicine. 2006; 34(3):44555. [DOI:10.1177/0363546505281241] [PMID]

[26] Pfile KR, Hart JM, Herman DC, Hertel J, Kerrigan DC, Ingersoll CD. Different exercise training interventions and drop-landing biomechanics in high school female athletes. Journal of Athletic Training. 2013; 48(4):450-62. [DOI:10.4085/1062-6050-48.4.06] [PMID] [PMCID] 